\title{
Ambulatory Blood Pressure Monitoring - Clinical Practice Recommendations
}

\author{
Mako Katalin*, Ureche Corina, Jeremias Zsuzsanna \\ University of Medicine and Pharmacy Tirgu Mures, Romania
}

Ambulatory blood pressure monitoring (ABPM) became a subject of considerable scientific interest. Due to the increasing use of the ABPM in everyday clinical practice it is important that all the users have a correct knowledge on the clinical indications, the methodology of using the device including some technical issues and the interpretation of results. In the last years several guidelines and position papers have been published with recommendations for the monitoring process, reference values, for clinical practice and research. This paper represents a summary of the most important aspects related to the use of ABPM in daily practice, being a synthesis of recommendations from the recent published guidelines and position papers. This reference article presents the practical and technical issues of ABPM, the use of this method in special situations, the clinical interpretation of measured values including the presentation of different ABPM patterns, derived parameters, the prognostic significance and the limitations of this method.

Keywords: ambulatory blood pressure monitoring, clinical interpretation, ABPM patterns, derived parameters

Received: 24 March 2016 / Accepted: 22 July 2016

\section{Introduction}

The initial evaluation of a patient with hypertension should confirm the diagnosis of hypertension, detect causes of secondary hypertension, and evaluate cardiovascular risk, target organ damage and concomitant clinical conditions. Ambulatory blood pressure monitoring has a considerable scientific interest with a large number of articles listed on PubMed in 2015. Due to the increasing use of the ABPM in everyday clinical practice, it is important for all the users (specialists, general practitioners) to have a correct knowledge about the clinical indications, the way to use this device and to interpret the results.

In the last years, several guidelines and position papers have been published with recommendations for the monitoring process, reference values, for clinical practice and research. In 2013, the European Society of Hypertension Working Group on BP Monitoring and Cardiovascular Variability published a position paper presenting the all aspects of this technique, based on the available scientific evidence for ABPM [1].

The latest NICE guidelines (2013), recommend the use of ABPM to confirm the diagnosis of hypertension if the clinic blood pressure is $140 / 90 \mathrm{~mm} \mathrm{Hg}$ or higher [2]. The Canadian Education Program in Hypertension also recommend ABPM or HBPM home monitoring during the second consultation for assessment of hypertension in patients without target organ damage, diabetes mellitus or chronic kidney disease but with BP lower than 180/110 $\mathrm{mm} \mathrm{Hg}$. Recent guidelines in hypertension management from the American Heart Association and from the Joint National Committee (JNC) 8 did not include specific comments on ABPM [3].

* Correspondence to: Katalin Mako

E-mail: makokatalin@yahoo.com
This article represents a schematic summary of the most important aspects related to the use of ABPM in daily practice, being a synthesis of the recommendations from the recent published guidelines and position papers [4-6].

\section{Indications for ambulatory blood pressure monitoring}

There are some advantages of ABPM over office BP measurement such as: a larger number of readings, profiles of $\mathrm{BP}$ behavior during the usual daily activity of the individual, reproducible mean day and night time values. ABPM has an important role in identifying masked and white-coat hypertension phenomenon, in evaluation of dipping patterns, nocturnal hypertension, BP variability throughout the day, assessment of the $24 \mathrm{~h}$ efficacy of antihypertensive medication. In conformity with the latest published studies, ABPM is a much stronger predictor of cardiovascular morbidity and mortality than office BP measurements or HBPM.

The indications of ABPM in clinical practice recommended by ESH Position Paper (2013) and Practice Guidelines Consensus Document (2014) are:

\section{Compelling indications:}

- Identifying white-coat hypertension phenomenon

- White-coat hypertension in untreated individuals

- White-coat effect in treated or untreated individuals

- False resistant hypertension due to white-coat effect in treated individuals

- Identifying masked hypertension phenomenon

- Masked hypertension in untreated individuals

- Masked uncontrolled hypertension in treated individuals

- Identifying abnormal 24h BP patterns 
- Daytime hypertension

- Siesta dipping/post-prandial hypotension

- Nocturnal hypertension

- Dipping status/isolated nocturnal hypertension

- Assessment of treatment

- Assessing 24h BP control

- Identifying true resistant hypertension

\section{Additional indications:}

- Assessing morning hypertension and morning BP surge

- Screening and follow up of obstructive sleep apnea

- Assessing increased BP variability

- Assessing hypertension in children and adolescents

- Assessing hypertension in pregnancy

- Assessing hypertension in the elderly

- Assessing hypertension in high-risk patients

- Identifying ambulatory hypotension

- Assessing endocrine hypertension

\section{Technical issues, satisfactory number of measurements}

The technique of ABPM: the patient wearing a portable blood pressure measuring device, preferably on the nondominant arm, for a period of 24 hours. This device gives information on BP during daily activities and at night during sleep. At the time of fitting of the portable device, the difference between the initial values and those from BP measurement by the physician should not be more than $5 \mathrm{mmHg}$, if the difference is greater than $5 \mathrm{mmHg}$, the cuff is recommended to be removed and replaced again. The examining physician trains the patients for performing normal activity during the ABPM. At the time of cuff inflation, the patient should stop moving and keep the arm still with the cuff at the level of the heart. The patient is asked to provide information in a diary on symptoms and events that may influence BP, in addition to the times of drug ingestion, meals, physical activity, and extreme stress.

The ABPM devices and software must correspond to some technical requirements and must be validated independently according to the internationally accepted validation protocols, which is essential for clinical use. The software should be able to provide a standardized plot format on one page, with different windows during the 24 hour period, identified and normal bands clearly demarcated showing the subject's awake and asleep time intervals. The data should include summary statistics for time-weighted systolic and diastolic BP and heart rate in the windows of the 24 hour period and separately for the awake and asleep periods (with standard deviations) and the number of valid $\mathrm{BP}$ readings included in the analysis.

Regarding the number of measurement for a satisfactory ABPM, it was considered acceptable to have a minimum of 20 valid daytime (awake) measurements and seven measurements at night (asleep), based on the requirement to have at least $70-80 \%$ of the measurements, obtained at least every $30 \mathrm{~min}$, or more frequently, throughout the entire 24 hour period.

\section{When to repeat ABPM}

The recommendation of repeating ABPM depends on a large number of factors and clinical situations. Some indications of repeated ABPM are: resistant hypertension, existence of comorbidities or target organ damages (diabetes, renal diseases, PAOD), family history of premature cardiovascular disease. Specific situations for repeating ABPM at relatively short intervals (3-6 months) are: the need to confirm the diagnosis of white-coat or masked hypertension, nocturnal hypertension, follow-up of high-risk patients. In cases of mild hypertension and low cardiovascular risk, ABPM may be repeated at 1 or 2-year intervals and regular home blood pressure monitoring is recommended for long-term follow-up.

\section{Special situations - difficult to perform ABPM}

Patients with atrial fibrillation: due to some special characteristics (increased beat-to-beat BP variability due to variations in ventricular filling time, stroke volume, and contractility) the blood pressure measurement in patients with atrial fibrillation is less accurate. There are no studies and published evidence regarding the utilization and the role of ABPM in patients with arrhythmias and, specifically with atrial fibrillation. Despite of these limitations there is no reason at the present time, to not perform ABPM in such patients. Larger trials in performing and evaluating patients on atrial fibrillation are needed.

Obese persons: The prevalence of hypertension and other specific comorbidities (obstructive sleep apnea, type 2 diabetes) is higher in obese patients and the ABPM is often needed for correct diagnosis of hypertension. In obese persons some technical difficulties, such as miscuffing may be present but these should not prevent them from undergoing this investigation. In such cases, when available, conically shaped cuffs with proper sizes should be used. Cuff dimensions are most important, and the appropriate cuff size according to arm diameter should be used (to cover $80-100 \%$ of the individual's arm circumference) and width ( $40 \%$ of the arm circumference). In patients with very large arms, the cuff can be placed on the forearm and the wrist must be kept at the level of the heart during measurement. Further investigations in the obese patients for a correct $\mathrm{ABPM}$ technique are required.

\section{Interpretation of ABPM, diagnosis of hypertension [7-9]}

\section{Daytime, night-time and 24-h blood pressure}

The average "awake" (day-time) and "asleep" (night-time) values and the mean 24 hours systolic and diastolic values are the most common parameters used in interpretation of ABPM. The night-to-day BP ratio represents the ratio between average night-time and daytime BP. BP normally decreases during the night - dipping phenomenon. Al- 
though the degree of night-time dipping has a normal distribution in a population setting, it is generally accepted that nocturnal BP presents a decrease of $>10 \%$ comparing to daytime values (night-day BP ratio $<0.9$ ). This is an arbitrary accepted cut-off value for definition of "dippers". Recently, more dipping categories have been proposed (see in „Patterns of diurnal variations of BP” section). Possible reasons for absence of dipping are: sleep disturbances, obesity, high salt intake, obstructive sleep apnea (OSAS), diabetic neuropathy (orthostatic hypotension, autonomic dysfunction), chronic kidney disease and old age.

\section{Threshold for hypertension diagnosis based on ABPM}

Definitions of hypertension by office and out-of-office blood pressure levels (ESC-ESH Guideline 2013) are:

- Office BP: systolic blood pressure (SBP) $\geq 140 \mathrm{mmHg}$ and/or diastolic blood pressure (DBP) $\geq 90$

- ABPM 24-h Average: SBP $\geq 130 \mathrm{mmHg}$ and DBP $>80 \mathrm{mmHg}$

- ABPM awake (daytime) average: $\mathrm{SBP} \geq 135 \mathrm{mmHg}$ and $\mathrm{DBP}>85 \mathrm{mmHg}$

- ABPM asleep (night-time) average: $\mathrm{SBP} \geq 120 \mathrm{mmHg}$ and $\mathrm{DBP}>70 \mathrm{mmHg}$

- Home BP: $\mathrm{SBP} \geq 135 \mathrm{mmHg}$ and/or $\mathrm{DBP}>85 \mathrm{mmHg}$

\section{BP variability}

BP being a dynamic parameter is characterized by continuous beat-to beat variations (very short term variability), within 24 hours (short term variability) and over the days, weeks or months (long term variability). The short-term $\mathrm{BP}$ variability can be evaluated with $\mathrm{ABPM}$, the longterm variability requires repeated $B P$ measurements over days, weeks with repeated measurements (office BP, home blood pressure monitoring or ABPM). The short-term BP variability might be considered for risk stratification in population and cohort studies, but currently it does not represent a parameter for routine use in clinical practice $[10,11]$. The proposed mechanisms influencing the short term variability are: increased central sympathetic drive, reduced arterial/cardiopulmonary reflex, reduced arterial compliance, humoral effects, rheological factors, emotional factors, behavioral influences/physical activity, sleep, postural changes. The clinical assessment of shortterm BPV has been based on calculation of the standard deviation (SD) of 24-hour average BP values, obtained through ABPM. Other newer ABPM parameters quantifying short-term BPV are: day-time/night time standard deviation (SD), coefficient of variation (CV), 24 hour weighted SD, residual BPV (remaining after exclusion of the slower components of the 24 hour BP profile through spectral analysis), average real variability (ARV - the average of the absolute differences between consecutive measurements). These newer parameters have been suggested to be better predictors of organ damage and cardiovascular risk than the conventional 24 hour SD $[10,11]$.

\section{Specific hypertension patterns White-coat hypertension}

The prevalence of white-coat hypertension is $20 \%$ to $25 \%$. Therefore, it is crucial to make a correct and accurate diagnosis and establish a correct therapeutic plan, which can be achieved using ABPM and home BP monitoring before prescribing antihypertensive therapy. The suspicion of white-coat hypertension is present when patients have high office BP values and normal BP out-of-office readings. One of the most important indications for ABPM in all international guidelines is to detect untreated patients with high office BP values, but normal readings during daily activities [12].

The traditional definition of white-coat hypertension is: elevated office blood pressure with a normal blood pressure during the awake period with ABPM. However, because of the contribution of asleep blood pressure as a predictor of outcome, it seems counterproductive to exclude this period from consideration. The 2013 ESH guidelines propose an alternative definition of white-coat hypertension, which encompasses subjects with office systolic/diastolic blood pressure readings of $\geq 140 / 90 \mathrm{~mm} \mathrm{Hg}$ and a 24 hour blood pressure $<130 / 80 \mathrm{~mm} \mathrm{Hg}$.

The definition of white-coat (or isolated office) hypertension is:

- untreated individuals with elevated office BP 140/90 $\mathrm{mmHg}$

- and 24h ABPM<130/80 mmHg/awake ABPM $<135 / 85 \mathrm{mmHg} / \mathrm{sleep}$ ABPM $<120 / 70 \mathrm{mmHg}$

- or Home $\mathrm{BP}<135 / 85 \mathrm{mmHg}$

The NICE guidelines recommend ABPM for all patients suspected of having hypertension because of a previously elevated office BP measurement, whereas the 2013 ESH guidelines recommend ABPM for the detection of whitecoat hypertension in low- or moderate - risk patients with elevated office BP. The ESH Position Paper recommends that "people with white-coat hypertension should have the diagnosis confirmed in 3 to 6 months and be followed at yearly intervals with ABPM, or home BP monitoring, so as to detect whether and when sustained hypertension occurs" [1].

\section{White coat effect}

The definition of white coat effect is: a certain elevation of $\mathrm{BP}$ regardless of the daytime ABPM values or the use of antihypertensive drugs that occurs in medical environment. The white-coat effect is present when the office BP is higher than the daytime mean ABPM measured BP values. Despite this, the white-coat hypertension is defined if the office $\mathrm{BP}$ is high, and the awake ABPM mean value is normal and the patient is not receiving any antihypertensive drug. The white-coat effect is a possible cause of false resistant hypertension and may be present in any hypertensive patient, regardless of the type and dose of antihypertensive drug taken [13]. 


\section{Masked hypertension}

The definition of masked hypertension is: normal office $\mathrm{BP}<140 / 90 \mathrm{mmHg}$ with elevated ABPM measured awake BP $(\geq 135 / 85 \mathrm{mmHg})$ or $\mathrm{HBP} \geq 135 / 85 \mathrm{mmHg}$. This form of hypertension should not be diagnosed in patients with antihypertensive medication; when treated subjects have a normal office BP, but persistently elevated ambulatory or home BP, the term masked uncontrolled hypertension should be used. Recent evidence suggests that masked uncontrolled hypertension is very often present on high-risk patients, and it is the isolated nocturnal hypertension as the clinical characteristic of these patients; therefore the major role of ABPM in the evaluation of patients with high cardiovascular risk must be highlighted again. The NICE guideline ignores to mention/highlight the usefulness of $\mathrm{ABPM}$ in detecting masked hypertension. The ESH/ESC 2013 guideline recommend ABPM for detecting masked hypertension in all subjects with high-normal office BP or normal office BP with asymptomatic organ damage or with high total cardiovascular risk.

\section{Patterns of diurnal variations of BP}

\section{Dipping:}

- Nocturnal systolic and diastolic BP fall $>10 \%$ of daytime values or

- Night/day systolic and diastolic BP ratio $<0.9$ and $>0.8$

- Normal diurnal systolic and diastolic BP pattern

\section{Reduced Dipping:}

- Nocturnal systolic and/or diastolic BP fall from 1 to $10 \%$ of daytime values or

- Night/day systolic and/or diastolic BP ratio $<1$ and $>0.9$

- Reduced diurnal systolic and/or diastolic BP pattern

- Associated with increased cardiovascular risk

\section{Non-dipping and rising:}

- No reduction or increase in nocturnal systolic and/or diastolic BP or

- Night/day systolic and/or diastolic BP ratio

- Associated with increased cardiovascular risk

\section{Extreme dipping:}

- Marked nocturnal systolic and/or diastolic BP fall $>20 \%$ of daytime systolic and/or diastolic values or

- Night/day systolic and/or diastolic BP ratio $<0.8$

- Debatable association with cardiovascular risk

\section{Nocturnal hypertension:}

- Increased absolute level of night time systolic and/or diastolic BP (120/70 mmHg)

- Associated with increased cardiovascular risk - may indicate obstructive sleep apnea

\section{Morning surge}

- Is defined as a rise in $\mathrm{BP}>55 \mathrm{~mm} \mathrm{Hg}$ from the lowest nighttime reading

- It presents a risk of stroke almost three times greater than that seen in patients without a morning elevation of BP

- Other cardiovascular events (e.g. myocardial infarction, angina pectoris) are more frequent in the morning hours than at other times of day

- Greater carotid intima-media thickness and circulating inflammatory markers coexist in hypertensive patients with a morning BP surge and this might contribute to the increased $\mathrm{CV}$ risk in these patients

\section{Nocturnal Phenomenon}

For assessing the blood pressure during the night (asleep values) $A B P M$ is the best measurement methodology. The definition dipping status is based on the BP curve from wakefulness to sleep, depending on whether BP falls, rises, or remains constant. In some patients, the nocturnal decrease of BP may be absent - this phenomenon is defined as nondipping status. In some cases, BP may even rise during the night sleeping hours to reach levels that are higher than daytime levels - this phenomenon is defined as reverse dipping or rising. In other cases, there may be a marked decrease of BP values during the night - this is the extreme dipping phenomenon. Morning surge represent the extent of the rise of $\mathrm{BP}$ values in the morning around the awakening period and this provides additional information about the cardiovascular risk and additional prognostic information about the patient.

A large amount of evidence was published about the prognostic superiority of nocturnal BP compared to daytime pressure in predicting outcome [14]. Isolated nocturnal hypertension (present in around of $7 \%$ of hypertensive patients) can be diagnosed only with ABPM. The dipping phenomenon (difference between awake and night-time $\mathrm{BP})$ has a normal distribution in a population, a nocturnal BP decrease $>10 \%$ of daytime values, corresponding to a night/day BP ratio $>0.9$ [15].

Due to the demonstrated unfavorable cardiovascular risk profile of nondippers recently, the ADA (American Diabetes Association) recommends the administration of more than one antihypertensive medication at bedtime. The ESH/ESC Position Paper considers the evidence supporting this recommendation as inadequate; the main purpose of antihypertensive treatment is the reduction of nocturnal hypertension and the achievement of a BP control during the 24-hour period [16].

\section{Additional analyses}

A number of additional parameters may be derived from $\mathrm{ABPM}$ recordings such as: $\mathrm{BP}$ variability, morning $\mathrm{BP}$ surge, blood pressure load, percent time elevation and the ambulatory arterial stiffness index. The predictive value of 
these indices has not yet clearly been determined; therefore they should be used as experimental added parameters, with no routine clinical use. Several of these indices are discussed in detail in ESH position papers and guidelines. The reference values of these ABPM derived indices and their possible clinical interpretation is listed in Table I.

\section{Limitations of ABPM}

Until recently, ABPM has been considered as being more expensive than other measurement techniques. However, it has been shown that ABPM is cost-effective, both in specialist services and in primary care. Unfortunately, ABPM remains under-used in Romania. Possible reasons may be the increased cost for patients, the limited availability of ABPM and the knowledge gap for many physicians about interpreting an ABPM recording versus a simple BP reading.

\section{Conclusions}

The superiority of ABPM to office BP in evaluating the risk for developing cardiovascular and renal events has been studied in a large spectrum of associated diseases and populations. Despite the extensive clinical use and an impressive number of scientific publications on this field, there are still some questions before ABPM can totally or partially replace office BP measurements for the diagnosis of hypertension and in establishing the of a correct therapeutic approach [17].

The superiority of ABPM has been shown in men and women, young and old, in untreated and treated hypertensive patients. A large number of data published regarding ABPM demonstrated that some specific markers of target organ damage on hypertensive patients (increased carotid intima media thickness, left ventricular hypertrophy, pulse wave velocity, ankle-brachial pressure index) are closely correlated with office BP values $[17,18]$. A large number of studies argument the superiority of ABPM versus office BP measurements: in the general population, in isolated systolic hypertension, in resistant hypertension, in patients with coronary artery disease, diabetes mellitus, and chronic kidney disease. All of these studies have obtained positive results with only one ABP monitoring at the beginning of the study, whereas the evaluated target organ damages (cardiovascular mortality, myocardial infarction, stroke, end-stage renal disease) occur years later $[9,19]$.
In clinical practice, the average of 24 hour, awake and asleep BP values are the recommended parameter for defining hypertension, for monitoring the antihypertensive treatment. Other derived parameters from ABPM have been considered to be relevant as BP variability, day/night ratio, morning surge, blood pressure load, percent time elevation, ambulatory arterial stiffness index. The potential clinical values of these derived parameters still remain to be confirmed because the value in risk stratification has not been proven yet.

Some studies have demonstrated that night-time BP values are a stronger predictor for cardiovascular additional risk than daytime values [14]. The night-day ratio is a significant predictor of cardiovascular outcome but, with little prognostic information above the average 24 hours $\mathrm{ABPM}$ values. The dipping pattern is the most consistent ABPM finding with a strong correlation with the incidence of cardiovascular events. Extreme dippers may have an increased risk for stroke, and the data on the increased cardiovascular risk of extreme dippers are in reduced number - the clinical significance of this phenomenon is uncertain [17]. Recent published studies have demonstrated that increased short-term and long-term BP variability is associated with the development, progression, and severity of cardiac, vascular, and renal damage and with an increased risk of cardiovascular events and mortality. The nondipping pattern is associated with different comorbidities, and is proposed to be produced by different mechanisms: baroreflex or autonomic dysfunction, abnormal sodium metabolism and nocturnal volume overload [20]. A particular characteristic of ABPM curve is the circadian variability and especially the morning surge which was demonstrated to be linked to vascular damage in the myocardium, large arteries and in the renal and cerebral circulation [21,22]. In the presence of the comorbidities such as diabetes, cardiac and renal disease, an increased risk for cardiovascular events, such as myocardial infarction and stroke, has also been described in patients with the presence of morning surge. For the evaluation of the clinical importance of short and long term variability, and also the role of the morning surge in cardiovascular risk pattern, further studies are required.

\section{Conflicts of interest}

The authors report no conflicts of interest.

Table I. The reference values of ABPM derived parameters and their possible clinical interpretation

\begin{tabular}{lll}
\hline Parameter & Reference value & Clinical interpretation \\
\hline Average BP/24 hr & $<125-130 / 80 \mathrm{mmHg}$ & $>135 / 85 \mathrm{mmHg}$ - diagnostic criteria for arterial hypertension \\
Average daytime BP & $<130-135 / 85 \mathrm{mmHg}$ & $>140 / 90 \mathrm{mmHg}$ - diagnostic criteria for arterial hypertension \\
Average daytime BP & $<120 / 70 \mathrm{mmHg}$ & $>125 / 75 \mathrm{mmHg}$ - diagnostic criteria for arterial hypertension \\
PP - pulse pressure & $<50 \mathrm{mmHg}$ & $>53 \mathrm{mmHg}-$ independent cardiovascular risc factor factor \\
Diurnal index - DI & $10-20 \%$ & $<10 \%$ non-dipper \\
& & $>20$ extreme dipping \\
Percent time elevation - PTE & $<15 \%$ & $>15 \%-$ diagnostic criteria for arterial hypertension \\
& & $>45 \%$ target organ damage \\
Blood pressure load or hyperbaric load & $<60 \mathrm{mmHg} \times$ hour & $>60 \mathrm{mmH}$ x hour - pressure overload \\
\hline
\end{tabular}




\section{References}

1. O'Brien E, Parati G, Stergiou G, et al. European Society of Hypertension Position Paper on Ambulatory Blood Pressure Monitoring. J Hypertens. 2013;31:1731-1767. doi: 10.1097/HJH.0b013e328363e964.

2. National Institute for Health and Clinical Excellence (NICE). Hypertension: the clinical management of primary hypertension in adults. Clinical Guideline 127.2011. www.nice.org.uk/guidance/CG127. 2013.

3. Chobanian AV, Bakris GL, Black HR, et al. Joint National Committee on Prevention, Detection, Evaluation, and Treatment of High Blood Pressure. National Heart, Lung, and Blood Institute; National High Blood Pressure Education Program Coordinating Committee. Seventh report of the Joint National Committee on Prevention, Detection, Evaluation, and Treatment of High Blood Pressure. Hypertension. 2003;42:1206-1252.

4. Turner JR, Viera AJ, Shimbo D. Ambulatory blood pressure monitoring in clinical practice: a review. Am J Med. 2015;128:14-20.

5. Weber MA, Turner JR. Ambulatory Blood Pressure Monitoring: New Directions and Uncertainties Arise From the U.S. Preventive Services Task Force Recommendation on the Diagnosis of Hypertension. $\mathrm{J}$ Clin Hypertens (Greenwich). 2016;18:172-174. doi: 10.1111/jch.12798.

6. O'Brien E, Dolan E, Atkins N. Failure to Provide ABPM to All Hypertensive Patients Amounts to Medical Ineptitude. J Clin Hypertens (Greenwich). 2015;17(6):462-465. doi: 10.1111/jch.12524.

7. Mancia G, Fagard R, Narkiewicz K, et al. List of authors Task Force Members.2013 ESH/ESC Guidelines for the management of arterial hypertension: The Task Force for the management of arterial hypertension of the European Society of Hypertension (ESH) and of the European Society of Cardiology (ESC). J Hypertens. 2013;31(7):1281-357. doi: 10.1097/01.hjh.0000431740.32696.cc.

8. O'Brien E, Parati G, Stergiou G. Ambulatory blood pressure measurement: what is the International Consensus? Hypertension 2013;62:988-994. doi: 10.1161/HYPERTENSIONAHA.113.02148.

9. Kikuya M, Hansen TW, Thijs L, et al. Diagnostic thresholds for ambulatory blood pressure monitoring based on 10-year cardiovascular risk. Circulation. 2007;115:2145-2152. DOI: 10.1161/ CIRCULATIONAHA.106.662254.

10. Clement DL. Blood pressure variability: proceedings of the International Workshop on Blood Pressure Variability held at the University Hospital. Ghent. UK: MTP Press Ltd. 2008.

11. Parati G, Onchoa JE, Lombardi C. Assessment and management of blood pressure variability. Nat Rev Cardiol. 2013;10;143-155. doi: 10.1038/nrcardio.2013.1.

12. O'Brien E, Atkins N, Stergiou G, et al. European Society of Hypertension International Protocol revision 2010 for the validation of blood pressure measuring devices in adults. Blood Press Monit. 2010:15:23-38. doi: 10.1097/MBP.0b013e3283360e98.

13. Parati G, Omboni S, Bilo G. Why is out-of-office blood pressure measurement needed? Home blood pressure measurements will increasingly replace ambulatory blood pressure monitoring in the diagnosis and management of hypertension. Hypertension. 2009; 54:181-187. DOI: 10.1161/HYPERTENSIONAHA.108.122853

14. Fan HQ, LiY, Thijs L. International Database on Ambulatory Blood Pressure In Relation to Cardiovascular Outcomes Investigators. Prognostic value of isolated nocturnal hypertension on ambulatory measurement in 8711 individuals from 10 populations. J Hypertens. 2010;28(10):2036-2045. doi: 10.1097/HJH.0b013e32833b49fe.

15. Rothwell PM, Howard SC, Dolan E, et al. Prognostic significance of visit-to-visit variability, maximum systolic blood pressure, and episodic hypertension. Lancet. 2010;375:895-905. doi: 10.1016/S01406736(10)60308-X.

16. Rothwell PM, Howard SC, Dolan E, et al. Effects of beta blockers and calcium-channel blockers on within-individual variability in blood pressure and risk of stroke. Lancet Neurol. 2010;9:469-480. doi: 10.1016/S14744422(10)70066-1.

17. Hansen TW, Thijs L, Li Y et al. Prognostic value of reading-to-reading blood pressure variability over 24 hours in 8938 subjects from 11 populations. Hypertension. 2010; 55:1049-1057. doi: 10.1161/ HYPERTENSIONAHA.109.140798.

18. O'Brien E, Asmar R, Beilin L, et al. European Society of Hypertension recommendations for conventional, ambulatory and home blood pressure measurement. J Hypertens. 2003; 21:821-848.

19. O'BrienE. Twenty-four-hour ambulatory blood pressure measurement in clinical practice and research: a critical review of a technique in need of implementation. J Intern Med. 2011;269:478-495. doi: 10.1111/j.13652796.2011.02356.x.

20. Dolan E, Stanton A, Thijs L, et al. Superiority of ambulatory over clinic blood pressure measurement in predicting mortality: the Dublin outcome study. Hypertension. 2005; 46:156-161. DOI: 10.1161/01. HYP.0000170138.56903.7a.

21. Metoki H, Ohkubo T, Kikuya M, et al. Prognostic significance for stroke of a morning pressor surge and a nocturnal blood pressure decline: the Ohasama study. Hypertension. 2006;47:149-154. DOI: 10.1161/01. HYP.0000198541.12640.0f.

22. Parati G, Stergiou GS, Asmar R, et al. European Society of Hypertension guidelines for blood pressure monitoring at home: a summary report of the Second International Consensus Conference on Home Blood Pressure Monitoring. J Hypertens. 2008;26:1505-1526. doi: 10.1097/ HJH.0b013e328308da66. 